\title{
Genetic heterogeneity in rapid onset dystonia- parkinsonism: description of a new family
}

\author{
K Kabakci, K Isbruch, K Schilling, K Hedrich, P de Carvalho Aguiar, L J Ozelius, P L Kramer, \\ M H R M Schwarz, C Klein
}

J Neurol Neurosurg Psychiatry 2005;76:860-862. doi: 10.1136/jnnp.2004.046730

\begin{abstract}
Rapid onset dystonia-parkinsonism (RDP) is a rare movement disorder with autosomal dominant inheritance, characterised by sudden onset of dystonic spasms and slowness of movement. To date, three families have been described that share linkage to the same location on chromosome 19q13, designated DYT12. Very recently, mutations in the ATPIA3 gene at the DYT12 locus have been demonstrated in seven unrelated patients, including the three previously linked families. A large RDP family is reported here, with eight definitely and one possibly affected members, that is not linked to the DYT12 region and has no mutation in the ATP1A3 gene. Predominant cranial-cervical involvement of dystonia occurred in this family, which has also been described in patients with idiopathic torsion dystonia linked to the DYT6 region on chromosome 8 and is a rare finding in DYT1 dystonia. Molecular genetic analysis also excluded linkage to the DYT6 locus and the GAG deletion in DYT1, suggesting at least one additional RDP gene.
\end{abstract}

$\mathrm{R}$ apid onset dystonia-parkinsonism (RDP) is an autosomal dominant movement disorder with reduced penetrance. RDP is characterised by sudden onset of orofacial dystonia, dysarthria, dysphagia, and involuntary dystonic spasms, predominantly of the upper limbs, along with signs of parkinsonism such as bradykinesia, rigidity, and postural instability. Symptoms usually manifest over hours to weeks and are followed by no or only moderate progression. Onset of symptoms is in adolescence or young adulthood. ${ }^{1-3}$

The RDP gene was mapped to an $8 \mathrm{cM}$ region on chromosome 19q13 (between markers D19S587 and D19S900; highest multipoint LOD score of 5.77 at D19S198) in two RDP families with 16 affected members. ${ }^{4}$ This locus was subsequently confirmed but not further refined in a third RDP family from Ireland with a detailed clinical description. $^{56}$ In addition, one apparently sporadic case from Spain was reported. ${ }^{7}$ Very recently, the RDP locus was refined to a $5.9 \mathrm{cM}$ region ${ }^{8}$ and six different mutations in the $\mathrm{Na}^{+} / \mathrm{K}^{+}$-ATPase $\alpha 3$ (ATPIA3) gene have been demonstrated in seven unrelated families, including all previously reported cases. ${ }^{9}$

Predominant cranial-cervical involvement of dystonia, as a characteristic feature of RDP, has also been described in patients with idiopathic torsion dystonia linked to the DYT6 region in two Mennonite families ${ }^{10}$ and is a rare finding in DYTl dystonia.

In this report we describe the clinical and genetic evaluation of a four generational German RDP family with eight definitely affected and one possibly affected members.

\section{METHODS \\ Patients}

After obtaining informed consent, all available family members underwent a detailed neurological examination, and videotaping was carried out on the index patient (III.15) and her mother (II.9). Information on deceased individuals was obtained by reviewing medical records or by interview of family members. The diagnosis of RDP was established according to the current clinical criteria and rated using the RDP rating scale. ${ }^{3}$ Clinical investigations of the index patient included routine laboratory testing, copper and caeruloplasmin analysis, EEG, and brain magnetic resonance imaging (MRI).

\section{DNA analysis}

The index patient was screened for the GAG deletion in DYTl. Linkage analysis was carried out on all available family members at the DYT12 and DYT6 loci using the following microsatellite markers:

\section{- DYT12: D19S224 (61.49cM) - D19S197 (63.10cM) - D19S223 (64.16cM) - D19S420 (66.30cM) - D19S900 (67.37cM) - D19S178 (68.08cM) - D19S574 (69.50cM) - D19S903 (69.50cM); \\ - DYT6: D8S1477 (60.34cM) - D8S1828 (71.00cM) - D8S1113 (77.89cM) - D8S1136 (82.26cM) - D8S2324 (94.08cM) - D8S1119 (101.01cM).}

Primer sequences were taken from the genome database (www.gdb.org). In addition, following the identification of the RDP gene at the DYT12 locus, we sequenced the ATP1A3 gene in our index patient as described. ${ }^{9}$

\section{Statistical analysis}

The computer program VITESSE ${ }^{11}$ was used for the LOD score analysis.

\section{RESULTS}

\section{Patients}

This German RDP family consists of 37 identified family members, 20 of whom could be examined, including all four living definitely affected members (fig 1). Information on the remaining 17 individuals was obtained by interview of relatives. Eight family members (four male, four female; mean (SD) age at onset, 7.1 (2.7) years, range 4 to 12 ) were definitely affected, five in generation II and three in generation III. In addition, individual I.2 (generation I) was affected by history and rated as possibly affected. Mode of inheritance appeared autosomal dominant.

No affected individual has been identified in generation IV as yet (current mean (SD) age, 17.3 (6.5) years; range 4 to 25). Of note, three individuals (II.5, II.6, and III.10) were

Abbreviations: RDP, rapid onset dystonia-parkinsonism 


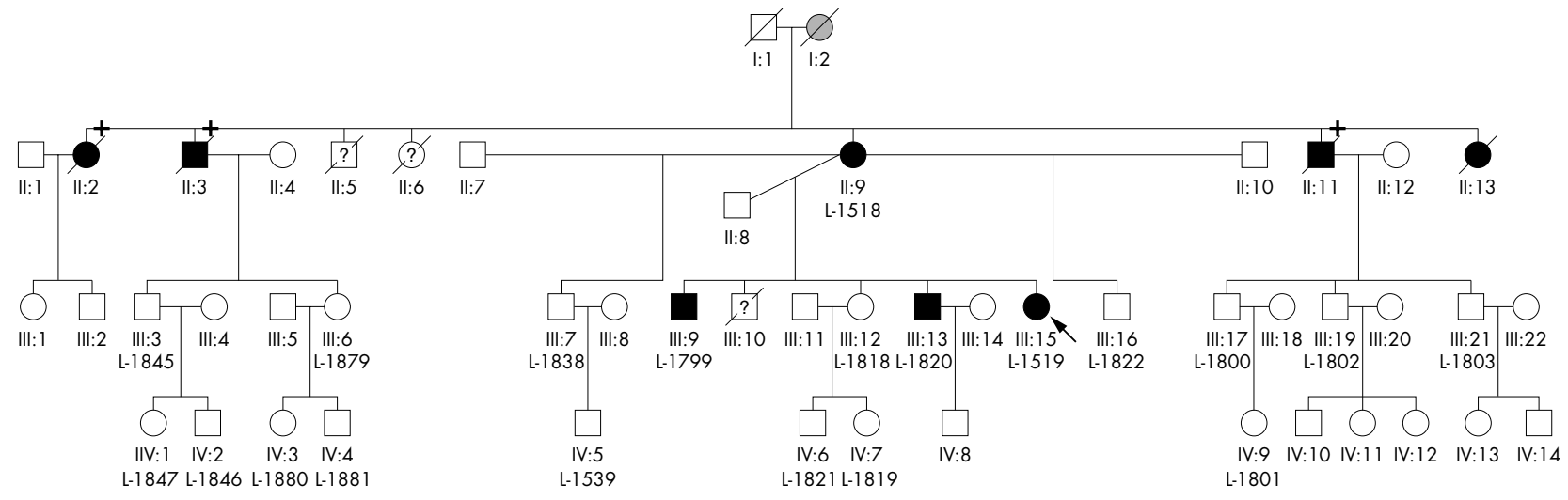

Figure 1 This four generational pedigree shows all eight definitely affected (black) and the possibly affected (grey) family members. The index patient is indicated by an arrow. The three individuals with a question mark died within their first year of life of unknown cause. The three affected members marked with a " + " had fatal renal failure. DNA for molecular genetic analyses was available for the 20 individuals with an " $L$ " number only.

born preterm and died of unknown cause in their first year of life. Three of the eight subjects with definite RDP developed renal failure with fatal outcome at ages of 33, 47, and 67 years, respectively (II.2, II.3, II.11). Very recently, the index patient (III.15) was diagnosed with hypoplastic kidneys at age 39 years. Her mother (II.9) has a history of renal cysts going back over several years.

The clinical features of the eight family members definitely affected by RDP are given in table 1. A short case report on the index patient is given below.

\section{Index case}

When aged six years, patient III.15 had acute onset (overnight) of difficulty in speaking and moving her tongue, along with dystonic spasms of the left hand, following febrile bronchitis. Symptoms rapidly progressed over the next few days. She developed severe dysphonia and dysphagia, requiring transient nasogastric feeding, orofacial dystonia, and dystonia of all four extremities. In addition, she showed an expressionless face, drooling, and generalised slowness of movement. Symptoms initially remained stable for about three weeks and were followed by partial remission. A levodopa trial was of no benefit. On neurological examination 33 years after disease onset, the patient showed severe dysarthria and generalised dystonia, most pronounced on the left hand side and in the upper body half, with predominant orofacial involvement. Parkinsonian signs had resolved with the exception of mild hypomimia and bradykinesia. The remainder of the neurological examination was normal. Routine laboratory tests, including copper and caeruloplasmin, were normal, as were an EEG and brain MRI. A lumbar puncture was not done.

\section{Molecular analysis}

The GAG deletion in DYTl was excluded in the index patient. Linkage analysis was carried out in all four living affected members and in the 16 unaffected members, and excluded the DYT12 and DYT6 loci on chromosomes 19q and 8p-q in this family. Specifically, all two point LOD scores for the chromosome 19q and 8p-q markers were $<-2.00$ (at $\theta=0.00$ ) and provide clear evidence against linkage in this family across both regions. In addition, mutations in the ATPIA3 gene were also excluded by direct sequencing of the coding region and exonintron boundaries in the index patient.

\section{DISCUSSION}

We describe the first family with RDP that is not caused by mutations in the ATPIA3 gene although it is clinically similar to the three previously described RDP pedigrees and to the
Spanish sporadic case, and conforms to the RDP diagnostic criteria. $^{2356}$ In particular, our family also showed a combination of dystonic and parkinsonian signs with abrupt or subacute onset, predominant bulbar involvement, and no response to levodopa treatment. RDP onset was preceded by a febrile illness in three of the eight definitely affected individuals in our family. Similarly, a trigger (stress/trauma) was reported in four of eight members of the Irish RDP family. ${ }^{5}$ Our family also confirms the intrafamilial variability of symptoms and disease course.

It should be borne in mind that the current RDP diagnostic criteria are based on only two RDP families. ${ }^{3}$ In this context, several distinguishing features of our family are worthy of note. First, the mean age of onset of about seven years was lower than in the previously described families, in whom the disease mostly started in adolescence. Surprisingly, no member of generation IV has become affected as yet. This may in part be explained by the fact that at least some individuals may not yet have reached the age of onset. Second, three family members were born preterm and died in their first year of unknown cause, and three of the eight definitely affected individuals had fatal renal failure. In addition, the index patient and her mother also suffer from kidney disease (table 1). The age of onset of this renal disorder was in adulthood and therefore it was not a trigger of their RDP. This suggests an as yet unidentified familial renal disorder; however, its potential relation to the RDP in this family remains elusive. Third, linkage to the RDP locus (DYT12 on chromosome 19q) and mutations in the ATPIA3 gene were excluded in our family.

Because of the predominant orofacial involvement in DYT6 dystonia that is somewhat reminiscent of the "bulbar dystonia" in RDP, we considered involvement of the DYT6 gene. This, however, also turned out to be negative. Owing to the early onset and broad spectrum of DYTI dystonia, we tested for the GAG deletion in the DYTl gene, and this was excluded as well.

Overall, our genetic results suggest the presence of at least one additional dominantly inherited gene causing RDP or a different disease mechanism in our family. The possibility of a mitochondrial (mtDNA) point mutation was investigated in a previous study but no disease causing mutation was found. ${ }^{3}$ As all affected individuals in our family inherited the disease from their mother, the mode of inheritance would be compatible with maternal transmission or with an autosomal dominant inheritance, possibly of a maternally expressed gene. This observation may thus warrant both analysis of the mtDNA and an investigation of the known paternally imprinted genes to identify a novel RDP gene in our family. 


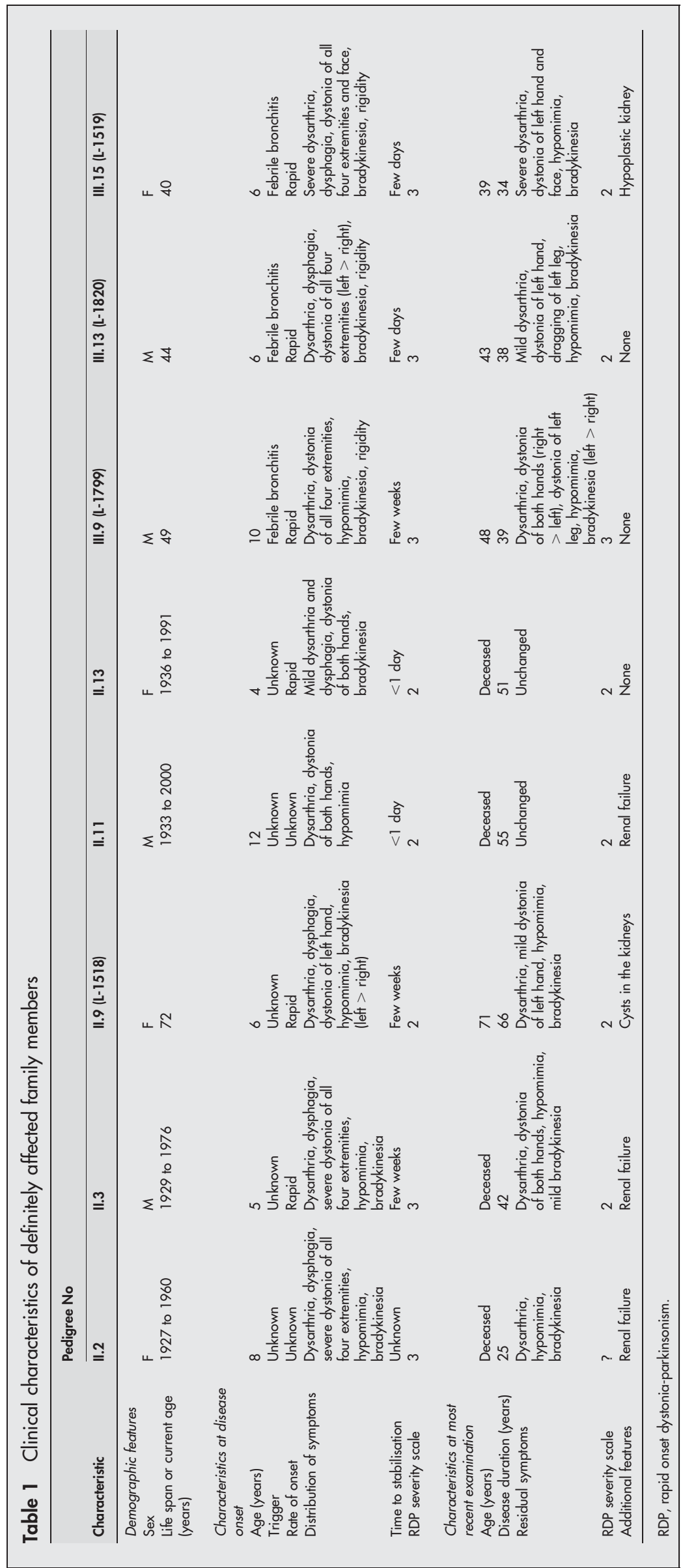

\section{ACKNOWLEDGEMENTS}

We would like to thank the family for participating in this study and Sylwia Dankert for technical support. This work was supported by the Deutsche Forschungsgemeinschaft (Kl-1134/3-1) and the Deutsche Dystonie Gesellschaft e. V.

\section{Authors' affiliations}

K Kabakci, K Hedrich, C Klein, Departments of Neurology and Human Genetics, University of

Lübeck, Lübeck, Germany

K Isbruch, M H R M Schwarz, Department of

Neurology, Klinikum Dortmund, Dortmund,

\section{Germany}

K Schilling, P L Kramer, Departments of Neurology, and Molecular and Medical Genetics, Oregon Health Sciences, University, Portland, Oregon, USA L J Ozelius, P de C Aguiar, Department of Molecular Genetics, Albert Einstein College of Medicine, New York, USA

Conflicting interests: none declared

Correspondence to: Dr Christine Klein, Department of Neurology, University of Lübeck, Ratzeburger Allee 160, 23538 Lübeck, Germany; klein_ch@ neuro.mu-luebeck.de

Received 2 June 2004

In revised form 9 September 2004

Accepted 22 September 2004

\section{REFERENCES}

1 Dobyns WB, Ozelius U, Kramer PL, et al. Rapid-onset dystonia-parkinsonism. Neurology 1993;43:2596-602.

2 Brashear A, Farlow MR, Butler IJ, et al. Variable phenotype of rapid-onset dystonia-parkinsonism. Mov Disord 1996;1 1:151-6.

3 Brashear A, Deleon D, Bressman SB, et al. Rapidonset dystonia-parkinsonism in a second family. Neurology 1997:48:1066-9.

4 Kramer PL, Mineta M, Klein C, et al. Rapid-onset dystonia-parkinsonism: linkage to chromosome 19q13. Ann Neurol 1999;46:176-82.

5 Pittock SJ, Joyce C, O'Keane V, et al. Rapid-onset dystonia-parkinsonism: a clinical and genetic analysis of a new kindred. Neurology 2000;55:991-5.

6 Webb DW, Broderick A, Brashear A, et al. Rapid onset dystonia-parkinsonism in a 14-year-old girl. Eur J Paediatr Neurol 1999:3:171-3.

7 Linazasoro G, Indakoetxea B, Ruiz J, et al. Possible sporadic rapid-onset dystonia-parkinsonism. Mov Disord 2002;17:608-9.

8 Kamm C, Leung J, Joseph S, et al. Refined linkage to the RDP/DYT12 locus on 19q13.2 and evaluation of GRIK5 as a candidate gene. Mov Disord:published online, 17 Mar, 2004.

9 de Carvalho Aguiar P, Sweadner KJ, Penniston JT, et al. Mutations in the $\mathrm{Na}+/ \mathrm{K}+-$ ATPase alpha3 gene ATP1A3 are associated with rapid-onset dystonia parkinsonism. Neuron 2004;43:169-75.

10 Almasy L, Bressman SB, Raymond D, et al. Idiopathic torsion dystonia linked to chromosome 8 in two Mennonite families. Ann Neurol 1997:42:670-3.

11 O'Connell JR, Weeks D. The VITESSE algorithm for rapid exact multilocus linkage analysis via genotype set-recoding and fuzzy inheritance. Nat Genet 1995; 11:402-8. 